\title{
Effect of aging on copper nanoparticles synthesized by pulsed laser ablation in water: structural and optical characterizations
}

\author{
R K SWARNKAR*, S C SINGH and R GOPAL \\ Laser Spectroscopy \& Nanomaterial Lab, Physics Department, University of Allahabad, Allahabad 211 002, India
}

MS received 23 June 2009

\begin{abstract}
Effect of aging on copper nanoparticles synthesized by pulsed laser ablation of copper plate in water was studied. By characterization studies of the aged nanoparticles, it is found that copper nanoparticles converted into $\mathrm{Cu} @ \mathrm{Cu}_{2} \mathrm{O}$ nanostructure. The synthesized nanomaterial is characterized with UV-Visible absorption, transmission electron microscopy (TEM), X-ray diffraction (XRD), Fourier transform infrared (FTIR) and Raman and photoluminescence (PL) spectroscopic techniques. TEM image shows that the aged nanoparticles get assembled into cactus like structure and are spherical in shape with average diameter $7 \mathrm{~nm}$ and dispersion $2 \mathrm{~nm}$. XRD and FTIR spectrum confirm the formation of $\mathrm{Cu} @ \mathrm{Cu}_{2} \mathrm{O}$ in the aged sample. Raman spectrum also confirms the presence of $\mathrm{Cu}_{2} \mathrm{O}$ nanoparticles. PL spectrum of the aged nanoparticles shows a direct allowed transition with bandgap energy of $2.24 \mathrm{eV}$. The mechanism for synthesis of core-shell nanoparticles and formation of self-assembly of nanoparticles is also discussed.
\end{abstract}

Keywords. $\mathrm{Cu} @ \mathrm{Cu}_{2} \mathrm{O}$ nanoparticles; laser ablation in aqueous medium; crystalline nanomaterials; optical characterization; nanostructured materials.

\section{Introduction}

Metal@metal oxide (core@shell) nanomaterials have shown considerable promise in the field of catalysis, sensing and optoelectronics etc. because they exhibit dual physical properties of core as well as shell materials. Metal oxides nanoparticles are used as base for gas sensors, whereas the addition of metallic core inside this oxide improves sensitivity and selectivity of the sensor (Aruna and Kruis 2007). The core@shell nanomaterials have many advantages, e.g. the ability to form self-assembly, optical tunability, solubility and reactivities involving optical, electronic, magnetic, catalytic, and chemical/biological phenomena (Zhong and Maye 2001; Lei and Chim 2005), etc which make them important.

Recently, copper-based nanomaterials are of great interest because of their optical, catalytical, mechanical and electrical properties, resulting in a wide range of applications in the field of metallurgy, catalysis, nanoand optoelectronics (Rakshani 1986; Cao et al 2003; Ji and Park 2007). $\mathrm{Cu}_{2} \mathrm{O}$ (cuprous oxide) and $\mathrm{CuO}$ (cupricoxide) are two important oxide compounds of copper and mostly behave like p-type semiconductor. Cuprous oxide is a II-VI semiconductor having direct bandgap of $\sim 2 \mathrm{eV}$, while that of cupric oxide has a monoclinic crys-

*Author for correspondence (rk_swarnkar85@rediffmail.com) tal structure with a bandgap of $1 \cdot 21-1 \cdot 51 \mathrm{eV}$ (Serin et al 2005). Copper oxide nanomaterials have lower surface potential barrier than metals, which affects their electron field emission properties, and therefore are highly applicable as electron sources in cathode ray tube and flat panel display (Hsieh et al 2003). Copper oxide and copper@copper oxide are considered as efficient catalytic agents (Samim et al 2007) and good gas-sensing (Barreca et al 2009) and biosensing (Umar et al 2009) materials and also play an important role in the optoelectronics (Hsieh et al 2008) and solar cell applications (Wu et al 2007). Nanoscaled $\mathrm{CuO}$ generated by thermal plasma technology (Ren et al 2009) containing traces of pure $\mathrm{Cu}$ and $\mathrm{Cu}_{2} \mathrm{O}$ shows activity against a range of bacterial pathogens, including meticillin-resistant Staphylococcus aureus (MRSA) and Escherichia coli. The ability of $\mathrm{CuO}$ nanoparticles to reduce bacterial populations to zero is enhanced in the presence of metal nanoparticles. There are a number of methods to synthesize the copper@ copper oxide nanomaterial like chemical vapour nucleation (Nasibulin et al 2001), template-based (Wen et al 2005), gas-solid reaction (Lin et al 2004), electrochemical (Borgohain et al 2000), hydrothermal (Gong et al 2006), RFsputtering (Ghodselahi et al 2008), etc. Laser ablation in liquid media is a 'top down' approach for the synthesis of nanomaterials having desired shape, size and chemical composition and surfaces free from chemical contamination, which are essential for further functionalization of nanomaterials for biological and sensing applications. 
This technique is adopted by several workers (Link et al 2000; Liang et al 2005; Sylvestre et al 2005; Singh and Gopal 2008; Singh et al 2009a) for the synthesis of colloidal solution of nanoparticles of different metals and semiconducting materials. Recently, adopting a similar method, our group has synthesized Zn@ZnO nanoparticles in aqueous medium of SDS (Singh et al 2010) using $1064 \mathrm{~nm}$ wavelength of pulsed Nd: YAG laser. The present paper deals with synthesis of copper and copper oxide nanoparticles by pulsed laser ablation in water and effect of aging on these nanoparticles.

\section{Experimental}

To produce the copper nanoparticles, a piece of copper metal (99.99\%, Spec-pure Johnson Mathey) placed at the bottom of glass vessel containing $20 \mathrm{ml}$ of double-distilled water (onward will be called simply water) was irradiated with the focused output of fundamental wavelength (1064 nm) of nanosecond pulsed Nd : YAG laser (Spectra Physics, USA) operating at $35 \mathrm{~mJ} /$ pulse energy, for $30 \mathrm{~min}$. A light green coloured colloidal solution was obtained after ablation.

The solution of so synthesized and aged colloidal nanoparticles was characterized by using optical and structural techniques. The UV-Visible absorption spectra of colloidal solution of nanoparticles so synthesized and after aging of 6 alternate days were acquired with Lambda 35, Perkin Elmer double beam spectrophotometer. The colloidal nanomaterial was dispersed onto a carbon-coated copper grid and dried for TEM analysis. High-resolution transmission electron microscopy (HRTEM) and TEM images of the nanoparticles after 30 days of aging were recorded on Technai G20-stwin electron microscope operating at $200 \mathrm{kV}$ with $1.44 \AA$ point and $2.32 \AA$ line resolutions. A part of colloidal solution of nanoparticles was centrifuged at $15000 \mathrm{rpm}$ and the obtained residue is dried at $60^{\circ} \mathrm{C}$. The XRD pattern of the dried powder was recorded after 15 days of aging using Rikagu, D-Max $\mathrm{X}$-ray diffractometer with $1.5406 \AA \mathrm{CuK} \alpha$ line. For recording the FTIR spectrum, the dried powder was dispersed into $\mathrm{KBr}$ matrix and palletized at a 10 ton pressure. This pellet was kept in the path of IR beam of Perkin Elmer RX-1 IR spectrometer, for recording the spectrum in the wavenumber region of $400-4000 \mathrm{~cm}^{-1}$. To record the photoluminescence (PL) and Raman spectra, colloidal solution of aged nanoparticles was taken in a $4 \mathrm{ml}$ quartz cuvette. The $514.5 \mathrm{~nm}$ and $488 \mathrm{~nm}$ lines of $\mathrm{Ar}^{+}$laser (Spectra Physics, Stabilite 2016) were used as excitation sources for PL and Raman, respectively. The spectra were recorded using computer controlled $0.5 \mathrm{M}$ triple grating monochromator (Acton Research Corp., USA) with PMT (R928, Hamamatsu) detector and $1800 \mathrm{~g} / \mathrm{mm}$ grating by using Spectra Sense software. Grams 32 software was used for spectral analysis and data processing.

\section{Results and discussion}

\subsection{UV-Visible absorption analysis}

The UV-Visible absorption spectrum of the as-synthesized solution of colloidal nanoparticles is recorded in the wavelength region of $200-800 \mathrm{~nm}$. It exhibits an intense peak centred at $\sim 270 \mathrm{~nm}$ and another peak with low intensity at $\sim 600 \mathrm{~nm}$ as shown in figure 1a. The peak at $\sim 270 \mathrm{~nm}$ is due to interband transition of copper electron from deep level of valence band while peak at $\sim 600 \mathrm{~nm}$ is due to interband transition of copper electron from upper level of valence band, which is also known as surface plasmon resonance (SPR) peak. The SPR of colloidal copper nanoparticles reported previously (Curtis et al 1988) with a peak at $590-640 \mathrm{~nm}$ is in agreement with the present result. With the passage of time the intensity of deeper interband and SPR transitions decreases rapidly as shown in figure $1 \mathrm{~b}$, which shows absorption spectra of colloidal solution of nanoparticles recorded on alternate days after the synthesis. Since the SPR peak is characteristic of the metal nanoparticles, intensity of SPR peak decreases with aging, which indicates oxidation of the produced nanoparticles taking place with time (Tilaki et al 2007). The broadening of SPR peak is due to the agglomeration of the nanoparticles in the sample and high width of their particle size distribution. As no capping agent is used, agglomeration of nanoparticles is fully expected. The blue shift in the peak centred at $\sim 270 \mathrm{~nm}$ in absorption spectra (figure $1 \mathrm{~b}$ ) may be due to the transition of electrons from the more inner shell of copper to the uppermost shell as time passes. It is possible that, due to aggregation and agglomeration, particle size increases and material settled down on the bottom of container causing decrease in the absorbance. But, aggregation and agglomeration will affect both the peaks. As deeper interband to SPR intensity ratio increases with time, i.e. SPR peak decreases faster with time than that of peak in UV region, indicating that oxidation overcomes the aggregation.

The optical bandgap of the as by synthesized nanoparticles is calculated using the Tauc relation (Singh et al 2009b) $\alpha h v=\left(h v-E_{\mathrm{g}}\right)^{n}$, where $h v$ is the incident photon energy and $n$ is the exponent that determines the type of electronic transition causing the absorption and can take the values $1 / 2,2 / 3,2$ and $3 / 2$. The best linear relationship is obtained by plotting $(\alpha h v)^{2}$ against $h v$, indicating that the optical bandgap of these nanoparticles is due to a direct allowed transition. Tauc plot for as synthesized colloidal nanoparticles is shown in figure 1c. The bandgap of as synthesized colloidal nanoparticles is determined from the intercept of the straight line at $\alpha=0$, which is found to be $2.39 \mathrm{eV}$.

\subsection{TEM analysis}

TEM image of the colloidal nanoparticles synthesized by pulsed laser ablation of copper in water recorded after an 

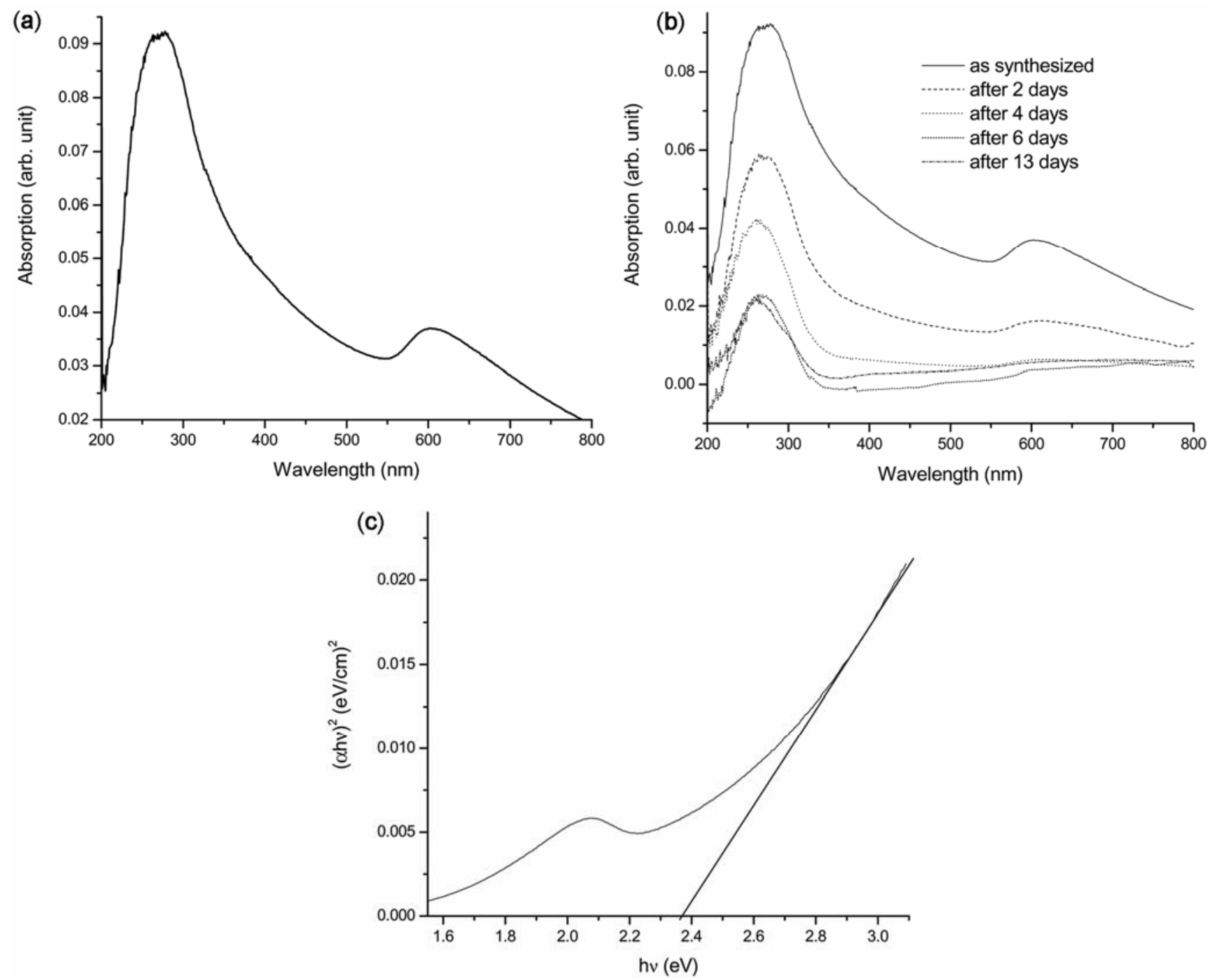

Figure 1. (a) UV-Visible absorption spectrum of as synthesized colloidal solution of nanoparticles by laser ablation of copper in water, (b) UV-Visible absorption spectra of colloidal nanoparticles recorded with aging of 6 alternate days and (c) Tauc plot of UVVisible absorption data of as synthesized colloidal nanoparticles for the calculation of bandgap energy.

Table 1. Estimated values of crystallite size and the microstrain calculated by using (1) and (2).

\begin{tabular}{lllcc}
\hline $2 \theta$ & Plane & Material & Crystallite size $(\mathrm{nm})$ & Microstrain \\
\hline $36.53^{\circ}$ & $(111)$ & $\mathrm{Cu}_{2} \mathrm{O}$ & 6.87799 & 0.01607 \\
$43.28^{\circ}$ & $(111)$ & $\mathrm{Cu}$ & 30.97814 & 0.00303 \\
$50.42^{\circ}$ & $(200)$ & $\mathrm{Cu}$ & 21.78156 & 0.00374 \\
$61.71^{\circ}$ & $(220)$ & $\mathrm{Cu}_{2} \mathrm{O}$ & 2.45581 & 0.02767 \\
$74.35^{\circ}$ & $(311)$ & $\mathrm{Cu}_{2} \mathrm{O}$ & 9.75241 & 0.0059 \\
\hline
\end{tabular}

aging of 30 days is displayed in figure $2 \mathrm{a}$ and HRTEM image in figure $2 \mathrm{~b}$. TEM image shows that aged nanoparticles are spherical in shape having average diameter of $7 \mathrm{~nm}$ and $2 \mathrm{~nm}$ dispersion and are self-assembled, forming cactus-like nanoarchitecture. The high resolution TEM image (shown in figure $2 b$ ) shows that the aged nanopar- ticles are not spherical but are elongated in shape. The value of inter-planer spacing of aged nanoparticles is calculated from HRTEM image and found to be $0.24 \mathrm{~nm}$ and $0.21 \mathrm{~nm}$, which correspond to the (111) and (200) planes of $\mathrm{Cu}_{2} \mathrm{O}$ (Salzemann et al 2004), respectively. HRTEM image also shows that the aged nanoparticles are not 
single crystals but consists of a number of randomly oriented small crystalline particles.

\subsection{XRD and FTIR analysis}

The XRD spectrum of the dried powder of nanoparticles was recorded after the aging of 15 days as shown in figure 3 . The XRD spectrum shows that the aged nanoparticles have two sharp peaks at $2 \theta=43 \cdot 28^{\circ}$ and $50.42^{\circ}$, which correspond to diffraction from the (111) and (200) planes of copper metal (JCPDS No. 04-0836), respectively, having cubical symmetry. In addition to these two sharp peaks there are three more broad peaks at $2 \theta=36.53^{\circ}, 61.71^{\circ}$ and $74.35^{\circ}$ corresponding to the (111), (220), and (311) planes of cubical $\mathrm{Cu}_{2} \mathrm{O}$ (Balamurugan and Mehta 2001) respectively. The XRD result confirms the presence of both copper and cuprous oxide in the aged sample of nanoparticles. This result is in agreement with UV-
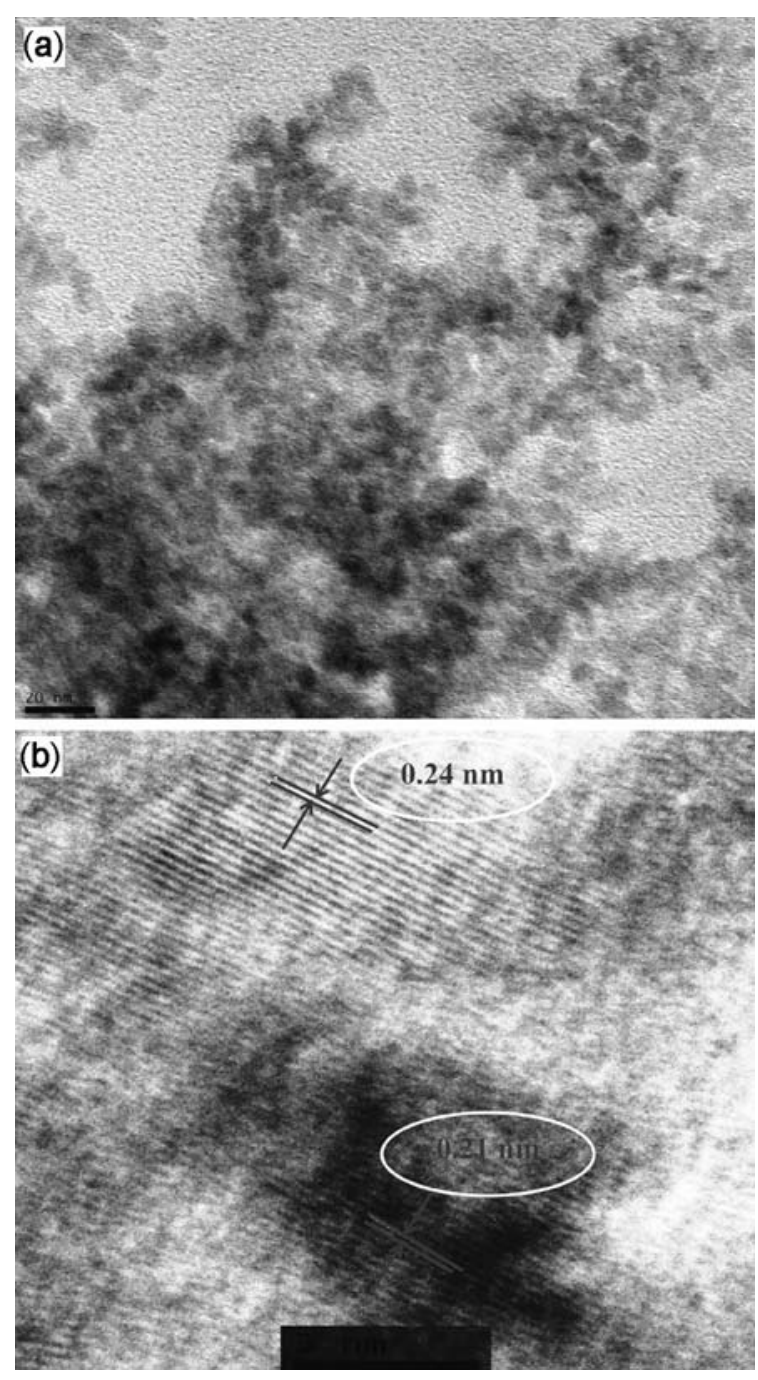

Figure 2. (a) TEM mictrographs of colloidal nanoparticles recorded after aging of 30 days. (b) HRTEM image of colloidal nanoparticles recorded after aging of 30 days.
Visible absorption result and the synthesized nanoparticles get oxidized with time and the copper nanoparticles converted in to $\mathrm{Cu} @ \mathrm{Cu}_{2} \mathrm{O}$. It is clear from the indexing of the nanoparticles that they have FCC structure. The existence of sharp peaks indicates that the synthesized nanoparticles are polycrystalline in nature. One can calculate the values of average crystallite size $(D)$ and microstrain $(\varepsilon)$ form XRD spectrum using the following equations:

$$
\begin{aligned}
& D=0.9 \lambda / \beta \cos \theta, \\
& \varepsilon=\beta / 4 \tan \theta,
\end{aligned}
$$

where $\lambda$ is the $\mathrm{X}$-ray wavelength, $\beta$ the full-width at halfmaximum of diffraction line in radian, and $\theta$ is half diffraction angle. The broadening of the diffraction line may be caused by either individual or combined effect of crystallite size, microstrain and crystal defects (stacking fraction). The broadening effect caused either by crystallite

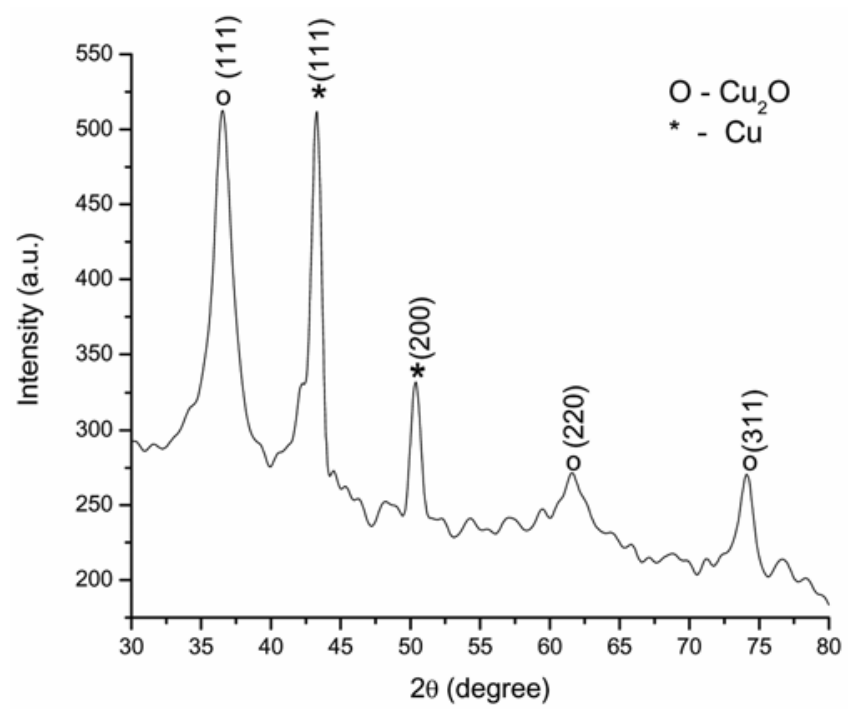

Figure 3. X-ray diffraction spectrum of nanoparticles recorded after aging of 15 days synthesized by laser ablation.

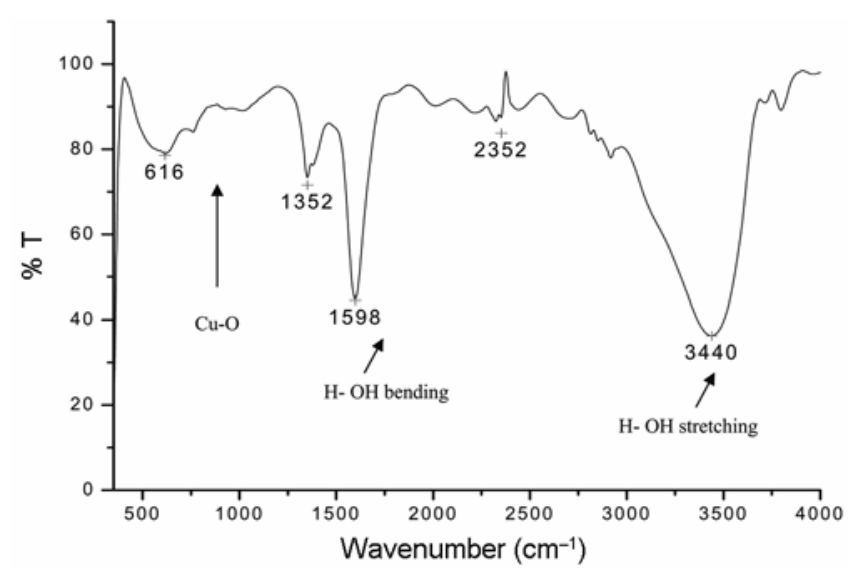

Figure 4. FTIR spectrum of aged nanoparticles synthesized by laser ablation. 
size or microstrain can be identified by examining the linearity of the $\beta \cos \theta$ vs $2 \theta$ and the $\beta \cot \theta$ vs $2 \theta$ curves. (1) is valid only if the crystallite shape is spherical (or approximately spherical) or polygonal in shape ( $\mathrm{Pu}$ et al 2008). The crystallite size calculated by using (1) and the value of microstrain calculated by using (2) corresponding to all the diffraction peaks are shown in table 1 . For $\mathrm{Cu}_{2} \mathrm{O}$, the crystallite size falls in the range $3-10 \mathrm{~nm}$, which is in agreement with TEM result.

The FTIR spectrum of aged nanopowder is shown in figure 4. An intense and wide peak centered at $3440 \mathrm{~cm}^{-1}$ is assigned as $\mathrm{H}-\mathrm{OH}$ stretching, and the peak at $1598 \mathrm{~cm}^{-1}$ corresponds to $\mathrm{H}-\mathrm{OH}$ bending. The peak centered at $615 \mathrm{~cm}^{-1}$ corresponds to the vibrational mode of $\mathrm{Cu}-\mathrm{O}$ in $\mathrm{Cu}_{2} \mathrm{O}$ phase (Heltemes 1966). Therefore, one can conclude that the aged nanoparticles have cuprous oxide.

\subsection{Raman spectroscopic analysis}

Raman activity is a function of the space group symmetry of a crystalline solid. It provides a wide range of information about the strength of interatomic and intermolecular bonds within the material, mechanical strain and the material's degree of crystallinity. The Raman shift can change, and bandwidth can broaden, with the decreasing particle size (Tanaka et al 1992, 1993). The Raman spectrum of aged colloidal solution of nanoparticles recorded after 40 days is shown in figure 5 . There is an intense and wide peak centered at $295 \mathrm{~cm}^{-1}$ is observed with a shoulder at $360 \mathrm{~cm}^{-1}$. These peaks correspond to the $2 \Gamma_{12}^{-}+\Gamma_{25}^{-}$ and $\Gamma_{2}$ mode of phonon vibration of $\mathrm{Cu}_{2} \mathrm{O}$ (Petroff et al 1975), respectively. A broad peak with less intensity centered at $640 \mathrm{~cm}^{-1}$ is also observed, which corresponds to the $\Gamma_{15}^{-(2)}$ mode. Raman analysis also confirms the

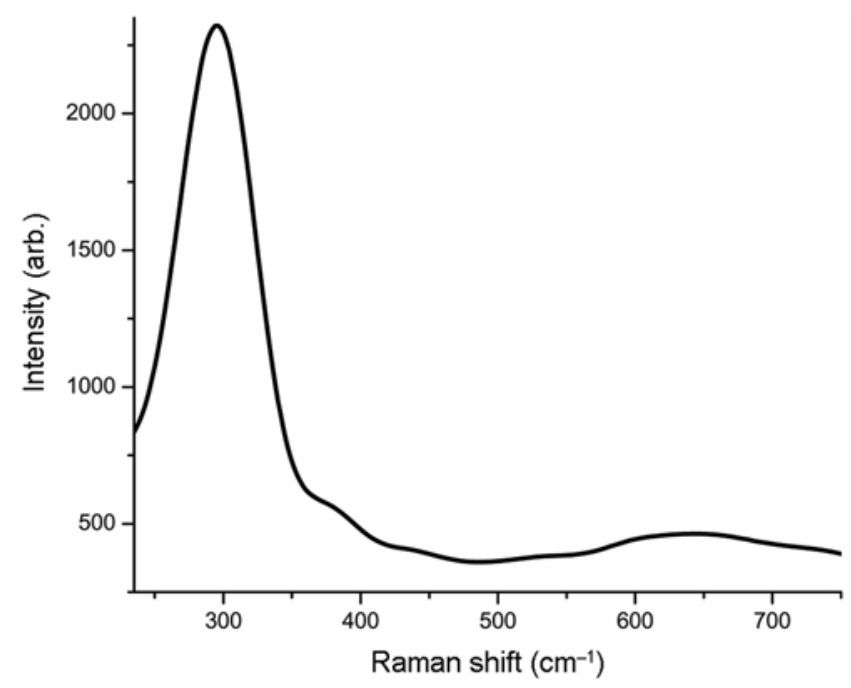

Figure 5. Raman spectrum of colloidal solution of nanoparticles synthesized by laser ablation of copper in water recorded after aging for 40 days. presence of $\mathrm{Cu}_{2} \mathrm{O}$ nanoparticles in aged colloidal solution, in agreement with the UV-Visible absorption, HRTEM, XRD and FTIR results.

\subsection{PL analysis}

The curve-fitted PL spectrum of nanoparticles synthesized in water recorded using $2.40 \mathrm{eV}(514.5 \mathrm{~nm})$ line of $\mathrm{Ar}^{+}$laser after 40 days of aging is shown in figure 6 . The spectrum shows a broad intense green luminescence having two peaks at $2 \cdot 24$ and $2 \cdot 14 \mathrm{eV}$. The peak at $2.24 \mathrm{eV}$ arises due to band to band transition of $\mathrm{Cu}_{2} \mathrm{O}$ nanoparticles, while the peak at $2.14 \mathrm{eV}$ is due to defects present inside the nanoparticles. The probable mechanism behind the PL phenomenon is sketched schematically in the figure 7. The valance band electrons absorb the light of $2.4 \mathrm{eV}(514.7 \mathrm{~nm})$ of $\mathrm{Ar}^{+}$laser and may excite to the conduction band. The transition of some electrons from the valance band to the conduction band emits green photons, corresponding to bandgap of the nanoparticles, while rest of the electrons transit to the defect levels by losing some of their energy by collisional relaxation. Transition of these electrons from defect levels close to the bottom of conduction band (donor level/electron trap level) to valance band or/and to the defect level (acceptor level/hole trap level), above the top of valance band, emits photons in the green regions. Jung and co-workers (Jung et al 2007) have studied the $\mathrm{PL}$ spectrum of $\mathrm{Cu}_{2} \mathrm{O}$ nanoparticles embedded in a polyimide layer and reported the peak at $\sim 2.26 \mathrm{eV}$ due to interband transitions from the ground electronic sublevel to the ground heavy-hole sublevel $\left(E_{1}-\mathrm{HH}_{1}\right)$ of the $\mathrm{Cu}_{2} \mathrm{O}$ nanoparticles. The defect level at $0.08 \mathrm{eV}$ is due to copper ion vacancies in cuprous oxide is also reported (Balamurugan et al 2002). The broadening

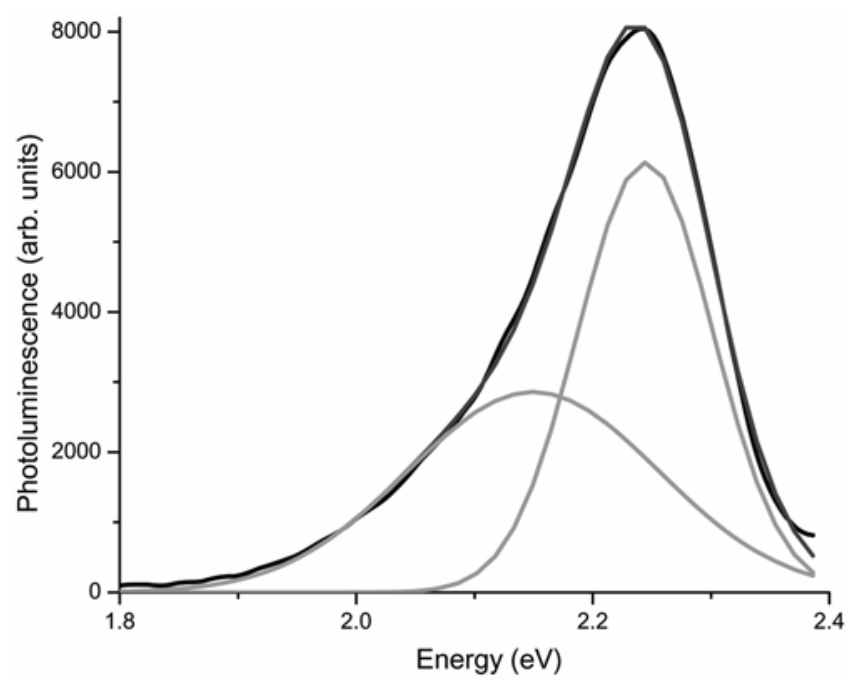

Figure 6. Photoluminescence spectrum of colloidal solution of nanoparticles synthesized by laser ablation of copper in water recorded after aging for 40 days. 


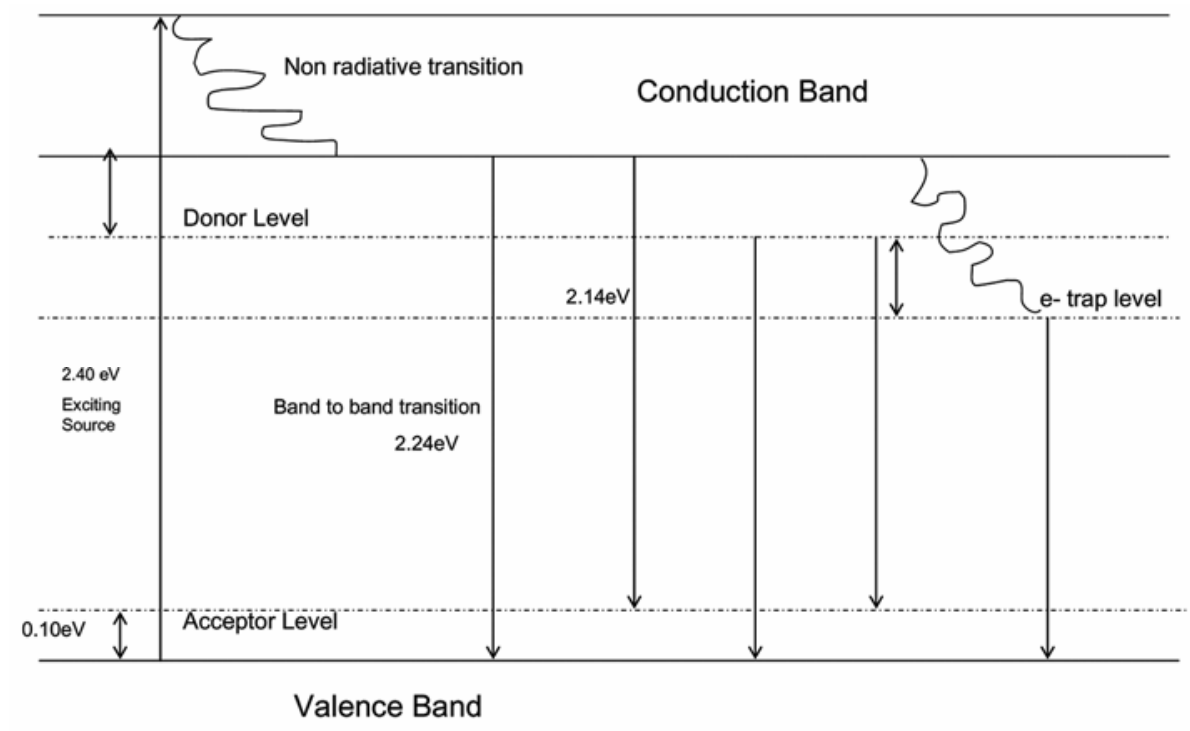

Figure 7. Schematic PL diagram.

of the PL band indicates that the synthesized nanoparticles are smaller in size, which is in agreement with the XRD and TEM results. The PL spectrum of the synthesized nanoparticles shows a broad peak in visible region; therefore, it can be used in the fabrication of green LEDs and optoelectronic devices.

\subsection{Mechanism for the synthesis of nanoparticles by} laser ablation in liquid medium, formation of $\mathrm{Cu} @ \mathrm{Cu}_{2} \mathrm{O}$ core-shell nanoparticles and self-assembly

Several researchers have already used the process of laser ablation to synthesize the nanoparticles of different metals and semiconductor materials in vacuum, gas and water media. Laser ablation of solids in a liquid medium has several advantages over ablation in vacuum or gas medium, e.g. the confinement of plume by liquid can greatly influence the thermodynamic and kinetic properties of the evolution of the plasma plume, and creates a different environment of the condensing phase formation from that of laser ablation of solids in vacuum or diluted gas. The mechanism of laser ablation in aqueous media can be understood as follows.

When laser light falls on the target surface, a part of the incident light is reflected and the rest is absorbed by the target surface, giving rise to thermal energy to cause heating, melting and vapourization of the target. The vapour also absorbs a portion of the incident laser beam, which raises the temperature and eventually leads to the formation of plasma. A re-solidification process takes place with the formation of the plasma, which results in particle formation within aqueous medium by coalescence.

Since copper is the highly oxidizing in nature, atoms on the surface of the nanoparticles get oxidized with the dissolved oxygen in the water, forming a thin layer of copper oxide on the surface of copper with time. As time passes, the copper atom in greater depth from the surface undergo oxidation and form a thicker shell of copper oxide on the surface of copper and form the copper@ copper oxide core@shell nanostructure. After a long time, the core of copper also gets oxidized and forms pure copper oxide nanoparticles. This explanation is strongly supported by UV-Visible absorption spectra, which shows decay and finally disappearance of the surface plasmon peak as time passes. On the basis of the above, the formation of $\mathrm{Cu}_{2} \mathrm{O}$ nanoparticles in the colloidal solution can take place according to the following equation

$$
\begin{aligned}
& 2 \mathrm{Cu} \text { (plasma) }+2 \mathrm{H}_{2} \mathrm{O} \rightarrow 2 \mathrm{Cu}(\mathrm{OH})+\mathrm{H}_{2}, \\
& 2 \mathrm{Cu}(\mathrm{OH}) \rightarrow \mathrm{Cu}_{2} \mathrm{O}+\mathrm{H}_{2} \mathrm{O} .
\end{aligned}
$$

The aged nanoparticles have a cactus-like structure as shown in figure $2 \mathrm{a}$, which is due to the self-assembly of the nanoparticles. The phenomenon of self-assembly formation can be understood as follows.

The reduction in particle size thermal fluctuation on its surface increase causes a thermal instability in the shape of nanoparticles. To gain a stable size/shape or structure with minimum thermal fluctuation, the nanoparticles get assembled and form several types of nanoarchitecture, such as cactus-like nanostructure, in our case. According to Lin et al $(2003,2004)$ the self-assembly of particles at fluid interfaces, driven by the reduction in interfacial energy, is well established. For the nanoparticles, thermal fluctuations compete with interfacial energy and give rise to a particle-size-dependent selfassembly. 


\section{Conclusion}

We have studied effect of aging on copper nanoparticles synthesized by pulsed laser ablation of copper metal in water and found that copper nanoparticles get oxidized and converted into $\mathrm{Cu} @ \mathrm{Cu}_{2} \mathrm{O}$ nanostructure. UV-Visible absorption spectra show that the as-synthesized nanomaterial is metallic in nature, which gets oxidized with time. The TEM image shows that the produced nanoparticles look like cactus-like structure due to self-assembly of nanoparticles with aging. The HRTEM image shows that the aged nanoparticles are crystalline in nature and are of $\mathrm{Cu}_{2} \mathrm{O}$. The oxidation of copper nanoparticles, with the passage of time, into $\mathrm{Cu}_{2} \mathrm{O}$ is also evident from XRD and FTIR spectra. The Raman shift at 295, 360 and $640 \mathrm{~cm}^{-1}$ are found, which correspond to the $2 \Gamma_{12}^{-}+\Gamma_{25}^{-}$, $\Gamma_{2}^{-}$and $\Gamma_{15}^{-(2)}$ mode of vibrations of $\mathrm{Cu}_{2} \mathrm{O}$, respectively. The PL spectrum of aged colloidal nanoparticles has a broad luminescence in the visible region and shows a direct allowed transition with bandgap energy of $\sim 2 \cdot 24 \mathrm{eV}$, corresponding to $\mathrm{Cu}_{2} \mathrm{O}$ nanoparticles.

\section{Acknowledgements}

The authors are thankful to Prof B R Mehta, IIT Delhi, New Delhi, Prof S B Rai, Banaras Hindu University, Varanasi, and NAC, Physics Department, Allahabad University, Allahabad, for providing TEM, FTIR and XRD facilities, respectively, and DRDO, New Delhi, for financial assistance.

\section{References}

Aruna I and Kruis F E 2007 Eur. Aerosol Conf. Salzburg Balamurugan B and Mehta B R 2001 Thin Solid Films 90396

Balamurugan B, Mehta B R, Avasthi D K, Singh F, Arora A K, Rajalakshmi M, Raghavan G, Tyagi A K and Shivaprasad S M 2002 J. Appl. Phys. 923304

Barreca D, Comini E, Gasparotto A, Maccato C, Sada C, Sberveglieri G and Tondello E 2009 Sensors and Actuators B: Chem. 141270

Borgohain K, Singh J B, Rao M V R, Shripati T and Mahamuni S 2000 Phys. Rev. B61 11093

Cao H S, Bonnet R, Hunsinger J J and Elkedim O 2003 Scripta Mater. 48531

Curtis A C, Duff D G, Edwards P P, Jefferson D A, Johnson B F G, Kirkland A I and Wallace A S 1988 J. Phys. Chem. 92 2211

Ghodselahi T, Vesaghi M A, Shafiekhani A, Baghizadeh A and Lameii M 2008 Appl. Surf. Sci. 2552730
Gong J, Luo L, Yu S, Qian H and Fei L 2006 J. Mater. Chem. 16101

Heltemes E C 1966 Phys. Rev. 803141

Hsieh C T, Chen J M, Lin H H and Shih H C 2003 Appl. Phys. Lett. 833383

Hsieh J H, Kuo P W, Peng K C, Liu S J, Hsueh J D and Chang S C 2008 Thin Solid Films $\mathbf{5 1 6} 5449$

Ji C and Park H S 2007 Nanotechnology 18305704

Jung J H, Kim T W, Song M S, Kim Y H and Yoo K H 2007 J. Appl. Phys. 101093708

Lei Y and Chim W K 2005 J. Am. Chem. Soc. 1271487

Liang C H, Shimizu Y, Sasaki T and Koshizaki N 2005 Appl. Phys. A80 819

Lin H, Wang C, Shih H C, Chen J and Hseish C $2004 \mathrm{~J}$. Appl. Phys. 955889

Lin Y, Skaff H, Emrick T, Dinsmore A D and Russell T P 2003 Science 226299

Link S, Burda C, Nikoobakht B and El-Sayed M A 2000 J. Phys. Chem. B104 6152

Nasibulin A G, Anonen P P, Richard O, Kauppinen E I and Altman I S 2001 J. Nanopart. Res. 3385

Petroff Y, Yu P Y and Shen Y R 1975 Phys. Rev. B12 2488

$\mathrm{Pu}$ Z, Yang C, Qin P, Lou Y and Cheng L 2008 Powder Diffraction 23213

Rakshani A E 1986 Solid State Electron. 729

Ren G, Hu D, Cheng E W, Vargas-Reus M A, Reip P and Allaker R P 2009 Int. J. Antimicrob. Agent 33587

Salzemann C, Lisiecki I, Brioude A, Urban J and Pileni M P 2004 J. Phys. Chem. B108 13242

Samim M, Kaushik N K and Maitra A 2007 Bull. Mater. Sci. 30 535

Serin N, Serin T, Horzum S and Celik Y 2005 Semicond. Sci. Tech. 20398

Singh S C and Gopal R 2008 J. Phys. Chem. C112 2812

Singh S C, Swarnkar R K and Gopal R 2009a J. Nanopart. Res. 111831

Singh S C, Swarnkar R K and Gopal R 2009b J. Nanosci. Nanotech. 95367

Singh S C, Swarnkar R K and Gopal R 2010 Bull. Mater. Sci. 3321

Sylvestre J P, Kabashin A V, Sacher E and Meunier M 2005 Appl. Phys. A80 753

Tanaka A, Onari S and Arai T 1992 Phys. Rev. B45 6587

Tanaka A, Onari S and Arai T 1993 Phys. Rev. B47 1237

Tilaki R M, Irajizad A and Mahdavi S M 2007 Appl. Phys. $\mathbf{A 8 8}$ 415

Umar A, Rahman M M, Al-Hajry A and Hahn Y B 2009 Electrochem. Commun. 11278

Wen X, Xie Y, Choi C L, Wan K C, Li X Y and Yang S 2005 Langmuir 214729

Wu C W, Wu J R and Chiang M J 2007 Electron devices and solid-state circuits, 673, DOI 10.1109/EDSSC.2007.4450215

Zhong C and Maye M M 2001 Adv. Mater. 131507 